\title{
Weakly countably determined spaces of high complexity
}

\author{
by
}

Antonio Avilés (Paris)

\begin{abstract}
We prove that there exist weakly countably determined spaces of complexity higher than coanalytic. On the other hand, we also show that coanalytic sets can be characterized by the existence of a cofinal adequate family of closed sets. Therefore the Banach spaces constructed by means of these families have at most coanalytic complexity.
\end{abstract}

1. Introduction. We deal with the descriptive complexity of a Banach space $X$ with respect to weak* compact subsets of the double dual $X^{* *}$. The simplest Banach spaces in this sense are reflexive spaces, which have a weakly compact ball and hence are $\mathcal{K}_{\sigma}$ (that is, $\sigma$-compact) subsets of the double dual. On the next level of complexity we find the class of Banach spaces which are $\mathcal{K}_{\sigma \delta}$ subsets (that is, countable intersections of $\mathcal{K}_{\sigma}$ sets) of their double dual, which includes all weakly compactly generated (WCG) spaces. Vašák [11] and Talagrand [9] introduced, respectively, the following two further descriptive classes:

Definition 1. A Banach space $X$ is called weakly countably determined (WCD) if there exists a family $\left\{K_{s}: s \in \omega^{<\omega}\right\}$ of weak* compact subsets of $X^{* *}$, and a set $A \subset \omega^{\omega}$, such that

$$
X=\bigcup_{a \in A} \bigcap_{n<\omega} K_{a \mid n} .
$$

Definition 2. A Banach space $X$ is called weakly $\mathcal{K}$-analytic (WKA) if there exists a family $\left\{K_{s}: s \in \omega^{<\omega}\right\}$ of weak* compact subsets of $X^{* *}$, and an analytic set $A \subset \omega^{\omega}$, such that

$$
X=\bigcup_{a \in A} \bigcap_{n<\omega} K_{a \mid n} .
$$

2000 Mathematics Subject Classification: 46B26, 54H05.

Key words and phrases: weakly countably determined Banach space, Gul'ko compact.

The author was supported by European Union Research Training Network PHD Marie Curie, Intra-European Fellowship MCEIF-CT2006-038768 and research projects MTM2005-08379 (MEC and FEDER) and Séneca 00690/PI/04 (CARM). 
Actually, in the case of WKA spaces, the analytic set $A$ can be chosen to be the whole Baire space $A=\omega^{\omega}\left({ }^{1}\right)$. Thus, the picture of the descriptive classes already considered in those works is the following:

$$
\mathrm{WCG} \subset \mathcal{K}_{\sigma \delta} \subset \mathrm{WKA} \subset \mathrm{WCD} .
$$

An important problem concerning this hierarchy is the "separation" problem, that is, constructing examples showing that the above inclusions are proper. To solve this problem, Talagrand used the technique of so-called adequate families of sets $\left({ }^{2}\right)$, which allowed him to produce two examples:

- A Banach space which is $\mathcal{K}_{\sigma \delta}$ but not weakly compactly generated [9].

- A weakly countably determined space which is not weakly $\mathcal{K}$-analytic [10].

The remaining separation problem was left open by Talagrand and has recently been solved by Argyros, Arvanitakis and Mercourakis [1], who provide an example of a WKA space which is not a $\mathcal{K}_{\sigma \delta}$ space. They construct their example by a technique different from Talagrand's adequate families, by using the so-called Reznichenko families of trees. Actually, their result shows that it is impossible to produce a $\mathcal{K}_{\sigma \delta}$ non-WKA space using adequate families, which explains Talagrand's failure to solve this question. Argyros, Arvanitakis and Mercourakis have also succeeded in showing that not only can the classes $\mathcal{K}_{\sigma \delta}$ and WKA be separated, but there is in fact a whole Borel hierarchy of spaces between them,

$$
\mathcal{K}_{\sigma \delta} \subset \mathcal{K}_{\sigma \delta \sigma \delta} \subset \cdots \subset \text { WKA. }
$$

In this note, we shall focus on higher levels of this hierarchy, on the gap between WKA and WCD. If one looks at Talagrand's example [10] separating these two classes, one realizes that the set $A \subset \omega^{\omega}$ which witnesses that the example is WCD is actually a complete coanalytic set. We propose the following definition:

Definition 3. Let $\mathcal{C}$ be a class of separable metrizable spaces. A Banach space is said to be weakly $\mathcal{C}$-determined if there exists a family $\left\{K_{s}: s \in\right.$ $\left.\omega^{<\omega}\right\}$ of weak* compact subsets of $X^{* *}$, and a set $A \in \mathcal{C}, A \subset \omega^{\omega}$, such that

$$
X=\bigcup_{a \in A} \bigcap_{n<\omega} K_{a \mid n}
$$

In this language, Talagrand's example [10] is a weakly $\Pi_{1}^{1}$-determined space which is not weakly $\Sigma_{1}^{1}$-determined (the symbols $\Pi_{1}^{1}$ and $\Sigma_{1}^{1}$ represent

$\left({ }^{1}\right)$ The reader can try to check this directly as an exercise, or else consider the characterizations of these concepts in terms of uscos [4, pp. 117-118, 142]; remember that any analytic set is a continuous image of $\omega^{\omega}$.

$\left({ }^{2}\right)$ Talagrand attributes the concept of adequate family to Roman Pol (cf. [9, p. 417]). 
the classes of coanalytic and analytic sets $\left({ }^{3}\right)$ in the logical notation, cf. [7]). The natural question arises: Are there WCD spaces of higher complexity? Namely, are there WCD spaces that are not weakly $\Pi_{1}^{1}$-determined? The two main results of this note are motivated by this problem and draw a similar picture as the one obtained by Argyros, Arvanitakis and Mercourakis on the lower level of the hierarchy.

We first analyze the technique of adequate families that Talagrand used for his two examples. We simplify this construction and show that the right framework for it is that of coanalytic sets. Again, although for different reasons than in the $\mathcal{K}_{\sigma \delta}$ problem, our Theorem 11 shows that Talagrand's technique cannot produce WCD spaces of higher complexity than coanalytic. This result is actually an intrinsic topological characterization of coanalytic sets which may be of independent interest.

Our second result states that the technique of Reznichenko families of trees developed in [1] does give a positive answer to our question: There are WCD spaces of complexity higher than coanalytic, actually there are WCD spaces of arbitrarily high complexity, in a sense that will be made precise. In particular, all projective classes can be separated:

TheOREM 4. For every $n \geq 1$ there exists a Banach space which is weakly $\Sigma_{n+1}^{1}$-determined but not weakly $\Sigma_{n}^{1}$-determined.

\section{General facts about WCD spaces}

Definition 5. A class $\mathcal{C}$ of separable metrizable spaces will be called nice if it is closed under the following operations:

- closed subspaces,

- continuous images,

- countable products,

- Wadge reduction, that is, if $f: A \rightarrow B$ is a continuous function between Polish spaces, $C \subset B, C \in \mathcal{C}$, then $f^{-1}(C) \in \mathcal{C}$.

Definition 6. Let $\Gamma$ be an uncountable set, let $K \subset \mathbb{R}^{\Gamma}$ be a compact subset, and $D$ a separable metrizable space. A mapping $f: \Gamma \rightarrow D$ is called a determining function if for every $x \in K$, every compact subset $C \subset D$, and every $\varepsilon>0$ the set $\left\{\gamma \in f^{-1}(C):\left|x_{\gamma}\right|>\varepsilon\right\}$ is finite.

We leave it to the reader to check that the fact that $f$ as above is a determining function is equivalent to any of the following two statements:

$\left({ }^{3}\right)$ It is usually said that a subset $A$ of a Polish space $X$ is Borel, analytic, coanalytic, $\Sigma_{n}^{1}, \Pi_{n}^{1}$, etc. However, all these properties are intrinsic topological properties of $A$ which do not depend on the Polish superspace [7]. Thus we talk about separable metrizable spaces which are Borel, analytic and so on. 
(i) For every $x \in K$ and $\varepsilon>0$ there is a neighborhood $V$ of $x$ in $K$ such that $\left\{\gamma \in f^{-1}(V):\left|x_{\gamma}\right|>\varepsilon\right\}$ is finite.

(ii) For every $x \in K$ and $\varepsilon>0$ the restriction of $f$ to the set $\{\gamma \in \Gamma$ : $\left.\left|x_{\gamma}\right|>\varepsilon\right\}$ is finite-to-one and has a closed and discrete range.

All the Banach spaces that we consider in this note are spaces $C(K)$ of continuous functions. The following theorem provides a useful criterion for $C(K)$ to be weakly $\mathcal{C}$-determined. The history of this result embraces [9], [6], [2], [8] and [5]. Originally it has been stated for WKA or WCD spaces, but it holds for any nice class $\mathcal{C}$.

Theorem 7. Let $\mathcal{C}$ be a nice class and let $K \subset \mathbb{R}^{\Gamma}$ be a compact set such that every $x \in K$ has countable support, that is, $\left|\left\{\gamma \in \Gamma: x_{\gamma} \neq 0\right\}\right| \leq \omega$. Then the following are equivalent:

(1) $C(K)$ is a weakly $\mathcal{C}$-determined space.

(2) There exists $D \in \mathcal{C}$ and a determining function $f: \Gamma \rightarrow D$.

Proof. First of all, in (2), $D$ can be taken to be a subset of $\omega^{\omega}$. The reason is that, because $\mathcal{C}$ is closed under Wadge reductions, for every $D_{1} \in \mathcal{C}$ there exists $D_{2} \subset \omega^{\omega}$ with $D_{2} \in \mathcal{C}$ such that $D_{2}$ maps continuously onto $D_{1}$, and we have the following fact:

Lemma 8. If $f: \Gamma \rightarrow D_{2}$ is a determining function and $g: D_{1} \rightarrow D_{2}$ is a continuous surjection, then there exists a determining function $f^{\prime}: \Gamma \rightarrow D_{1}$.

Proof of the lemma. Choose any mapping $s: D_{2} \rightarrow D_{1}$ (not necessarily continuous) such that $g s=1_{D_{2}}$. We prove that $s f: \Gamma \rightarrow D_{1}$ is a determining function. Indeed, otherwise there would exist $x \in K, \varepsilon>0$ and $C \subset D_{1}$ compact such that $\left\{\gamma \in(s f)^{-1}(C):\left|x_{\gamma}\right|>\varepsilon\right\}$ is infinite. But

$$
\left\{\gamma \in(s f)^{-1}(C):\left|x_{\gamma}\right|>\varepsilon\right\} \subset\left\{\gamma \in f^{-1}(g(C)):\left|x_{\gamma}\right|>\varepsilon\right\} .
$$

This contradicts the assumption that $f$ is a determining function.

After this observation, the statement of the theorem is the same as $[5$, Theorem 10(c)] after changing " $K$ is Talagrand compact" to " $C(K) \in \mathcal{C}$ ", " $\Gamma=\bigcup_{\sigma \in \omega^{\omega}} \bigcap_{j=1}^{\infty} \Gamma_{\sigma \mid j}$ " to " $\Gamma=\bigcup_{\sigma \in D} \bigcap_{j=1}^{\infty} \Gamma_{\sigma \mid j}$ for some $D \in \mathcal{C}$ ", and " $\forall \sigma \in \omega^{\omega}$ " to " $\forall \sigma \in D$ ". It is now a long but straightforward exercise that the proofs of [5, Theorems 4 and 10(c)], with obvious modifications, yield the assertion of Theorem 7.

The compact spaces $K$ for which $C(K)$ is WCG, WKA and WCD are called Eberlein, Talagrand and Gul'ko compact respectively. We define $\mathcal{C}$ Gul'ko compact spaces to be those compact spaces $K$ for which $C(K)$ is weakly $\mathcal{C}$-determined.

3. Adequate families on coanalytic sets. A family $\mathcal{A}$ of subsets of a set $X$ is called adequate if it has the following two properties: 
- If $A \in \mathcal{A}$ and $B \subset A$, then $B \in \mathcal{A}$.

- If $B \subset X$ is such that all finite subsets of $B$ belong to $\mathcal{A}$, then $B \in \mathcal{A}$.

In other words, to say that $\mathcal{A}$ is an adequate family is equivalent to saying that a subset $B \subset X$ belongs to $\mathcal{A}$ if and only if every finite subset of $B$ belongs to $\mathcal{A}$. Every adequate family of subsets of $X$ can be naturally viewed as a closed subset of the product $\{0,1\}^{X}$, and hence is a compact Hausdorff space.

A case of interest for us occurs when $X$ is a separable metrizable space and $\mathcal{A}$ is an adequate family of closed subsets of $X$ (actually closed and discrete, since the family is hereditary), because then we get a weakly countably determined space:

Theorem 9. Let $\mathcal{C}$ be a nice class, $X \in \mathcal{C}$, and $\mathcal{A}$ an adequate family of closed subsets of $X$. Then $C(\mathcal{A})$ is a weakly $\mathcal{C}$-determined Banach space.

This follows immediately from Theorem 7 , just taking the identity $f$ : $X \rightarrow X$ as a determining function. Talagrand's example from [9] is an adequate family of closed subsets of $X=\omega^{\omega}$, and the one from [10] is an adequate family of closed subsets of $X=\mathrm{WF}$, the set of well founded trees on $\omega^{<\omega}$, the standard complete coanalytic set. The fact that the former Banach space is WKA and the latter is WCD follows immediately from the above theorem. But the negative part, that they are not WCG and WKA respectively, needs further arguments and relies on the fact that these adequate families are big enough (of course, not any adequate family of closed sets would work). We have isolated the property of these adequate families that makes them as complicated as their underlying set.

Definition 10 . We say that an adequate family $\mathcal{A}$ of closed subsets of a topological space $X$ is cofinal if for every infinite closed and discrete subset $B$ of $X$ there exists an infinite subset $A \subset B$ such that $A \in \mathcal{A}$.

The following theorem is the main result of this section. The implication $(2) \Rightarrow(1)$ is a generalization and at the same a simplification of Talagrand's construction from [10] (in particular, we avoid any manipulation with trees, using instead the easier and more general coanalytic structure). The converse $(1) \Rightarrow(2)$ establishes the impossibility of performing this construction outside the framework of coanalytic sets.

TheOREM 11. For a separable metrizable space $X$ the following are equivalent:

(1) There exists a cofinal adequate family of closed subsets of $X$.

(2) $X$ is coanalytic.

Proof. Let $(K, d)$ be a compact metric space which contains $X$ as a dense set, $K=\bar{X}$, and let $Y=K \backslash X$. We denote by $\mathcal{M}$ the space of all 
strictly increasing sequences of positive integers, which is homeomorphic to the Baire space $\mathbb{N}^{\mathbb{N}}$.

$(1) \Rightarrow(2)$. Let $\mathcal{A}$ be a cofinal adequate family of closed subsets of $X$ and let $\left\{a_{n}: n=1,2, \ldots\right\}$ be an enumeration of a dense subset of $X$. We consider the set

$C=\left\{(y, \sigma) \in K \times \mathcal{M}:\left\{a_{\sigma_{1}}, a_{\sigma_{2}}, \ldots\right\} \in \mathcal{A}\right.$ and $d\left(y, a_{\sigma_{i}}\right) \leq \frac{1}{i}$ for all $\left.i \geq 1\right\}$.

In order to prove that $X$ is coanalytic, we check that $Y$ is analytic by showing that $C$ is a closed subset of $K \times \mathcal{M}$ and $Y=\{y \in K: \exists \sigma \in \mathcal{M}$ with $(y, \sigma) \in C\}$. If we pick $(y, \sigma) \in K \times \mathcal{M} \backslash C$ then either $\left\{a_{\sigma_{1}}, a_{\sigma_{2}}, \ldots\right\} \notin \mathcal{A}$ or there exists $i \in \mathbb{N}$ such that $d\left(y, a_{\sigma_{i}}\right)>1 / i$. In the former case, since $\mathcal{A}$ is an adequate family, there exists $j \in \mathbb{N}$ such that $\left\{a_{\sigma_{1}}, \ldots, a_{\sigma_{j}}\right\} \notin \mathcal{A}$ and then $\left\{(z, \tau) \in K \times \mathcal{M}: \tau_{r}=\sigma_{r} \forall r \leq j\right\}$ is a neighborhood of $(y, \sigma)$ which does not intersect $C$. In the latter case, there exists a neighborhood $U$ of $y$ such that $d\left(z, a_{\sigma_{i}}\right)>1 / i$ for all $z \in U$ and then $\left\{(z, \tau): z \in U\right.$ and $\left.\tau_{i}=\sigma_{i}\right\}$ is a neighborhood of $(y, \sigma)$ which does not intersect $C$. This proves that $C$ is closed.

We now show that $Y=\{y \in K: \exists \sigma \in \mathcal{M}:(y, \sigma) \in C\}$. Fix $y \in Y$. The sequence $\left\{a_{1}, a_{2}, \ldots\right\}$ is dense in $X$ which is dense in $K$, so there exists a subsequence $\left\{a_{n_{1}}, a_{n_{2}}, \ldots\right\}$ which converges to $y$. Since $y \notin X$, the set $\left\{a_{n_{1}}, a_{n_{2}}, \ldots\right\}$ is closed and discrete in $X$, hence, as $\mathcal{A}$ is a cofinal adequate family in $X$, this sequence has a subsequence $\left\{a_{m_{1}}, a_{m_{2}}, \ldots\right\} \in \mathcal{A}$ which still converges to $y$. We can pass to a further subsequence $\left\{a_{k_{1}}, a_{k_{2}}, \ldots\right\} \in \mathcal{A}$ such that $d\left(a_{k_{i}}, y\right) \leq 1 / i$. If we set $\sigma=\left(k_{1}, k_{2}, \ldots\right)$, we find that $(y, \sigma) \in C$. Conversely, suppose that $(y, \sigma) \in C$. Then $\left\{a_{\sigma_{1}}, a_{\sigma_{2}}, \ldots\right\}$, being a member of the adequate family $\mathcal{A}$, is a closed and discrete subset of $X$, but at the same time converges to $y$, so $y \notin X$, and hence $y \in Y$.

$(2) \Rightarrow(1)$. Suppose that $C \subset K \times \mathcal{M}$ is a closed set such that $Y=$ $\{x \in K: \exists \sigma \in \mathcal{M}$ with $(x, \sigma) \in C\}$. Let $\prec$ be a well order on $X$ (the use of the axiom of choice here is not essential, but it permits avoiding a number of technicalities in the proof). We define a cofinal adequate family $\mathcal{A}$ in the following way. A finite set belongs to $\mathcal{A}$ if and only if it is of the form $\left\{x_{1} \prec \cdots \prec x_{n}\right\}$ and there exists $(y, \sigma) \in C$ such that $d\left(y, x_{i}\right) \leq 1 / \sigma_{i}$ for every $i=1, \ldots, n$ (notice that this is a hereditary condition: if a finite set satisfies it, then so does every subset). An infinite set belongs to $\mathcal{A}$ if and only if every finite subset belongs to $\mathcal{A}$.

First, we show that every infinite set $A \in \mathcal{A}$ is closed and discrete in $X$. Otherwise, there would exist a sequence $\left\{x_{1} \prec x_{2} \prec \cdots\right\} \subset A$ which converges to a point $x \in X$ with $x \neq x_{i}$ for all $i$. Since $A \in \mathcal{A}$, for every $n$ there exists $\left(y^{n}, \sigma^{n}\right) \in C$ such that $d\left(y^{n}, x_{i}\right) \leq 1 / \sigma_{i}^{n}$ for every $i \leq n$. Notice that $\left(y^{n}\right)$ also converges to $x$ because $d\left(y^{n}, x_{n}\right) \leq 1 / \sigma_{n}^{n} \leq 1 / n$ (recall 
that all sequences in $\mathcal{M} \subset \omega^{\omega}$ are strictly increasing). Observe also that for every $i \in \omega$ the sequence $\left\{\sigma_{i}^{n}: n=1,2, \ldots\right\}$ is eventually constant with ultimate value that we call $\sigma_{i}^{\infty}$ (otherwise $\left(y^{n}\right)$ would converge to $x_{i}$, since $d\left(y^{n}, x_{i}\right) \leq 1 / \sigma_{i}^{n}$ for every $\left.n \geq i\right)$. The sequence $\left\{\sigma^{1}, \sigma^{2}, \ldots\right\}$ converges in $\mathcal{M}$ to $\sigma^{\infty}=\left(\sigma_{1}^{\infty}, \sigma_{2}^{\infty}, \ldots\right)$, and also $\left(y^{n}, \sigma^{n}\right)$ converges to $\left(x, \sigma^{\infty}\right)$; since $C$ is closed, we find that $\left(x, \sigma^{\infty}\right) \in C$, contrary to $x \in X$.

It remains to show that $\mathcal{A}$ is cofinal in $X$. We select an infinite closed and discrete subset $B$ of $X$. Viewing $B$ as a subset of the compact space $K$, we know that there exists a sequence $\left\{x_{1} \prec x_{2} \prec \cdots\right\} \subset B$ which converges to a point $y \in K$. Since $B$ is closed and discrete in $X$, it must be the case that $y \in Y$. Therefore, we can pick $\sigma \in \mathcal{M}$ such that $(y, \sigma) \in C$ and then we can find a subsequence $\left\{x_{n_{1}} \prec x_{n_{2}} \prec \cdots\right\}$ such that $d\left(y, x_{n_{i}}\right) \leq 1 / \sigma_{i}$ for every $i$. This subsequence is an element of $\mathcal{A}$, since every finite cut of the sequence satisfies the definition of $\mathcal{A}$ with the same witness $(y, \sigma) \in C$.

We devote the rest of this section to checking that for a cofinal adequate family $\mathcal{A}$ of closed subsets of a separable metrizable space $X$, the complexity of $C(\mathcal{A})$ is the same as that of $\mathcal{A}$. We mention that Čižek and Fabian [3] already realized that, by transferring the original examples of Talagrand, given any 0-dimensional complete metrizable space $X$, for every coanalytic non-Borel subset $Y \subset X$ there is an adequate family of subsets of $Y$ such that the corresponding compact space is Gul'ko but not Talagrand compact, and that for every Borel non- $\sigma$-compact subset $Y \subset X$ there is an adequate family of subsets of $Y$ such that the corresponding compact space is Talagrand but not Eberlein compact. They also gave a simpler approach to checking the negative part in the first kind of examples, which we shall follow.

For a family $\mathcal{A}$ of subsets of a set $X$ and a subset $Z \subset X$, we denote by $\left.\mathcal{A}\right|_{Z}=\{A \cap Z: A \in \mathcal{A}\}$ the restriction of $\mathcal{A}$ to $Z$. When $\mathcal{A}$ is an adequate family we can write $\left.\mathcal{A}\right|_{Z}=\{A \in \mathcal{A}: A \subset Z\}$.

TheOrem 12. Let $X$ be a coanalytic space, $\mathcal{A}$ a cofinal adequate family of closed subsets of $X$, and $Z$ a subset of $X$. Then $\left.\mathcal{A}\right|_{Z}$ is an Eberlein compact if and only if $Z$ is contained in some $\sigma$-compact subset of $X$.

Corollary 13. Let $X$ be a Borel non- $\sigma$-compact space, $\mathcal{A}$ a cofinal adequate family of closed subsets of $X$, and $Z \subset X$ any subset not contained in any $\sigma$-compact subset of $X$. Then $\left.\mathcal{A}\right|_{Z}$ is a Talagrand non-Eberlein compact space.

We notice that it follows from [1, Theorem 1.4] that if an adequate family $\mathcal{A}$ is Talagrand compact, then indeed $C(\mathcal{A})$ is a $\mathcal{K}_{\sigma \delta}$ space.

Proof of Theorem 12. Assume $\left.\mathcal{A}\right|_{Z}$ is Eberlein compact. $Z$ being metrisable and separable, every set in $\left.\mathcal{A}\right|_{Z}$ is at most countable. Hence, there is 
a decomposition $Z=\bigcup_{n<\omega} Z_{n}$ such that for every $n<\omega$ the family $\left.\mathcal{A}\right|_{Z_{n}}$ contains finite sets only [4, Theorem 4.3.2]. Fix $n<\omega$; we show that $Z_{n}$ is a relatively compact subset of $X$. Let $\left(z_{m}\right)_{m<\omega}$ be a one-to-one sequence in $Z_{n}$, and suppose for contradiction that it contains no subsequence convergent in $X$. Then it must contain a subsequence which is closed and discrete in $X$. From the cofinality of $\mathcal{A}$ this subsequence contains an infinite subset $A \in \mathcal{A}$. Hence $A \cap Z_{n}$ is infinite, a contradiction.

Conversely, suppose that $Z \subset \bigcup_{n<\omega} K_{n}$ where each $K_{n}$ is a compact subset of $X$. Fix any $A \in \mathcal{A}$ and any $n<\omega$. We claim that the set $A \cap Z \cap K_{n}$ is finite. If not, because $A$ is closed and $K_{n}$ is compact, this set would contain a sequence convergent to some $x \in K_{n} \cap A$, contrary to the discreteness of $A$. Having proved the claim, we conclude that $\left.\mathcal{A}\right|_{Z}$ is Eberlein compact by [4, Theorem 4.3.2].

Theorem 14. Let $X$ be a coanalytic space, $\mathcal{A}$ a cofinal adequate family of closed subsets of $X$, and $Z$ a subset of $X$. Then $\left.\mathcal{A}\right|_{Z}$ is Talagrand compact if and only if $Z$ is contained in some Borel subspace of $X$.

Corollary 15. Let $X$ be a coanalytic non-Borel space, $\mathcal{A}$ a cofinal adequate family of closed subsets of $X$, and $Z$ any subset of $X$ not contained in any Borel subspace of $X$. Then $\left.\mathcal{A}\right|_{Z}$ is Gul'ko (in fact $\Pi_{1}^{1}-G u l ' k o$ ) compact, but not Talagrand compact.

Proof of Theorem 14. If $Z$ is contained in some Borel space $B \subset X$, then $\left.\mathcal{A}\right|_{Z}$ can be viewed as an adequate family of closed subsets of $B$, and then it follows from Theorem 9 that $C\left(\left.\mathcal{A}\right|_{Z}\right)$ is WKA.

Now suppose that $\left.\mathcal{A}\right|_{Z}$ is Talagrand compact. Then by Theorem 7 there is a determining function $f: Z \rightarrow A$ with $A$ an analytic set; actually, there is a determining function $\psi: Z \rightarrow \omega^{\omega}$ (compose $f$ with a selection for a continuous surjection $\omega^{\omega} \rightarrow A$ ). We consider as usual a compact metric space $K$ with $\bar{X}=K$. For a finite sequence $\left(k_{1}, \ldots, k_{n}\right)$ of natural numbers we define $\left[k_{1}, \ldots, k_{n}\right]=\left\{\tau \in \omega^{\omega}: \forall i \leq n \tau_{i}=k_{i}\right\}$. We claim that

$$
Z \subset \bigcup_{\sigma \in \omega^{\omega}} \bigcap_{n \in \omega} \overline{\psi^{-1}\left[\sigma_{1}, \ldots, \sigma_{n}\right]} \subset X,
$$

where the closures are taken inside $K$. The first inclusion is clear since $z \in \bigcap_{n \in \omega} \overline{\psi^{-1}\left[\left(\psi(z)_{1}, \ldots, \psi(z)_{n}\right)\right]}$ for every $z \in Z$. For the second inclusion, suppose by contradiction that for some $\sigma \in \omega^{\omega}$ we have $y \in$ $\bigcap_{n \in \omega} \overline{\psi^{-1}\left[\sigma_{1}, \ldots, \sigma_{n}\right]} \backslash X$. Then we can find a sequence of elements $y_{n} \in$ $\psi^{-1}\left[\sigma_{1}, \ldots, \sigma_{n}\right]$ which converges to $y$. Since $y \notin X,\left\{y_{n}: n \in \omega\right\}$ is an infinite closed and discrete subset of $X$, so by cofinality we find a subsequence $a=\left\{y_{n_{k}}: k \in \omega\right\} \in \mathcal{A}$. Then the image of the support of $\left.a \in \mathcal{A}\right|_{Z}$ under $\psi$ is a convergent sequence in $\omega^{\omega}$, contrary to $\psi$ being a determining function. 
Thus $Z$ is contained in a subset of $X$ which is a Suslin operation on closed subsets of $K$, hence analytic. Since $X$ is coanalytic, by the separation theorem (any two disjoint analytic sets in a Polish space can be separated by disjoint larger Borel sets) we deduce that $Z$ is contained in a Borel subspace of $X$.

4. Gul'ko compact spaces of higher complexity. We now recall the notion of Reznichenko family of trees associated to a hereditary family of sets and the corresponding compact space, which have been introduced and studied in [1]. In what follows, by a tree we mean a set $T$ endowed with a partial order relation $\leq$ such that (1) for every $t \in T$ the set $\{s \in T: s<t\}$ is well ordered, and (2) $T$ has a $\prec$-minimum, called the root of $T$. An element of the tree $t \in T$ is called a node of $T$. An immediate successor of $t \in T$ is a node $s<t$ for which there is no further node $r$ with $t<r<s$. For an ordinal $\alpha$, the $\alpha$ th level of the tree $T$ is the set of all $t \in T$ such that $\{s: s<t\}$ has order type $\alpha$. The height of a tree is the first ordinal $\alpha$ for which the $\alpha$ th level is empty. A subset $S$ of a tree $(T, \leq)$ is a segment if (1) any two elements of $S$ are $\leq$-comparable and (2) if $t, s \in S, r \in T$ and $t \leq r \leq s$ then $r \in S$. A segment $S$ is initial if it contains the root of $T$.

Let $A$ be a set of cardinality at most $\mathfrak{c}$, and $\mathcal{F}$ a hereditary family of subsets of $A$ (if $B \in \mathcal{F}$ and $C \subset B$, then $C \in \mathcal{F}$ ). An $(A, \mathcal{F})$-Reznichenko family of trees is a family $\left\{T_{a}: a \in A\right\}$ of trees indexed by $A$ with the following properties:

(1) For every $a \in A, T_{a}$ is a tree of height $\omega$ in which every node has $\mathfrak{c}$ immediate successors (in particular, $T_{a}$ has cardinality $\mathfrak{c}$ ).

(2) $T_{a} \cap A=\{a\}$ and $a$ is the root of $T_{a}$.

(3) For every $t \in \bigcup_{a \in A} T_{a}$, we have $\left\{a \in A: t \in T_{a}\right\} \in \mathcal{F}$.

(4) For any $a, b \in A, a \neq b$ and any segments $S \subset T_{a}$ and $S^{\prime} \subset T_{b}$, we have $\left|S \cap S^{\prime}\right| \leq 1$.

(5) For every $B \in \mathcal{F}$ and every disjoint family $\left\{S_{b}: b \in B\right\}$ where $S_{b}$ is a finite initial segment of $T_{b}$ for every $b \in B$, there exist $\mathfrak{c}$ elements $t$ that are immediate successors of $S_{b}$ in $T_{b}$ simultaneously for all $b \in B$.

In this context we put $\mathcal{T}=\bigcup_{a \in A} T_{a}$ and let $R[\mathcal{F}] \subset 2^{\mathcal{T}}$ denote the family of all segments of all the trees $T_{a}$, which can be easily checked to be a compact family. It is shown in [1] that for any given set $A$ of cardinality $\mathfrak{c}$ and any hereditary family $\mathcal{F}$ of subsets of $A$ there exists an $(A, \mathcal{F})$-Reznichenko family of trees.

Recall that $\omega^{<\omega}$ is the set of finite sequences of natural numbers, ordered in the following way: $\left(s_{i}\right)_{i<n}<\left(t_{i}\right)_{i<m}$ if $n \leq m$ and $s_{i}=t_{i}$ for $i<n$. In order to avoid confusion with the concept of tree introduced before, we define a tree on $\omega$ to be a subset $T \subset \omega^{<\omega}$ such that if $a \in T$ and $b<a$ then $b \in T$. 
We denote by $\operatorname{Tr} \subset 2^{\omega^{<\omega}}$ the family of all trees on $\omega$; this is a compact family and is viewed as a compact metrizable space. A branch of $T \in \operatorname{Tr}$ is an infinite sequence $a \in \omega^{\omega}$ such that $a \mid n \in T$ for all $n$.

We fix $A \subset \mathcal{N}=\omega^{\omega}$. For every $s \in \omega^{<\omega}$ we define $W_{s}=\{a \in A: s \prec a\}$ where $s \prec a$ means that if $s=\left(s_{i}\right)_{i<n}$, then $s_{i}=a_{i}$ for all $i<n$. These sets constitute a basis for the topology of $A$. Also, we denote by $\operatorname{wf}(A) \subset \operatorname{Tr}$ the family of all trees on $\omega$ none of whose branches are elements of $A$. The following theorem asserts that in this context, a compact space $R[\mathcal{F}]$ constructed as above from a hereditary family of closed and discrete subsets of $A$ is always Gul'ko compact, with complexity bounded by that of wf $(A)$. This is nothing else than a more informative restatement of some lemmas from [1]. Nevertheless, we include a complete proof.

Theorem 16. Let $A \subset \mathcal{N}$ and $\mathcal{T}=\bigcup_{a \in A} T_{a}$, let $\mathcal{F}$ be a hereditary family of closed and discrete subsets of $A$, and let $R[\mathcal{F}] \subset 2^{\mathcal{T}}$ be the compact set coming from an $(A, \mathcal{F})$-Reznichenko family of trees. Then there exists a determining function $f: \mathcal{T} \rightarrow \operatorname{wf}(A) \times \omega^{\left(\omega^{<\omega}\right)}$.

Proof. Let $t \in \mathcal{T}=\bigcup_{a \in A} T_{a}$, and let $B(t)=\left\{a \in A: t \in T_{a}\right\}$, which is a set from $\mathcal{F}$ and hence closed and discrete in $A$. We define

$$
f_{1}(t)=\left\{s \in \omega^{<\omega}:\left|W_{s} \cap B(t)\right|>1\right\} .
$$

Clearly, $f_{1}(t) \in \operatorname{wf}(A)$ because if $a \in A$ were a branch of $f_{1}(t)$ then $a$ would be a cluster point of $B(t)$, and this contradicts the fact that $B(t)$ is closed and discrete.

On the other hand, for every $a \in B(t)$ we define $s_{a}^{t}$ to be the lowest element $s \in \omega^{<\omega}$ such that $s \prec a$ and $s \notin f_{1}(t)$. We define a function $f_{2}: \mathcal{T} \rightarrow \omega^{\left(\omega^{<\omega}\right)}$ in the following way: for $a \in B(t), f_{2}(t)\left(s_{a}^{t}\right)$ equals the level of the tree $T_{a}$ in which $t$ lies; if $s$ is different from any $s_{a}^{t}$, then set $f_{2}(t)(s)=0$. Thus, we have defined a function $f: \mathcal{T} \rightarrow \operatorname{wf}(A) \times \omega^{\left(\omega^{<\omega}\right)}$ by $f(t)=\left(f_{1}(t), f_{2}(t)\right), t \in T$. It remains to show that it is a determining function.

Let $C \subset \operatorname{wf}(A) \times \omega^{\left(\omega^{<\omega}\right)}$ be compact and suppose by contradiction that there is an element $x \in R[\mathcal{F}] \subset 2^{\mathcal{T}}$, that is, a branch $x=\left\{t_{1}<_{a} t_{2}<_{a} \cdots\right\}$ of the tree $T_{a}$ for some $a \in A$, such that $f(x) \subset C$. Two cases arise:

CASE 1: The elements $s_{a}^{t_{n}}$ are equal to some fixed $s \in \omega^{<\omega}$ for infinitely many $n$ 's. For these $n$ 's we have $n \leq f_{2}\left(t_{n}\right)\left(s_{a}^{t_{n}}\right)=f_{s}\left(t_{n}\right)(s)$, which contradicts $f(x) \subset C$ with $C$ compact.

CASE 2: Modulo passing to a subsequence, we may assume that $s_{a}^{t_{1}}<$ $s_{a}^{t_{2}}<\cdots \prec a$. For every $n$ consider the element $u_{n}<s_{a}^{t_{n}}$ which has length one less than $s_{a}^{t_{n}}$. Then $u_{n} \in f_{1}\left(t_{n}\right)$, and the $u_{n}$ 's show that $a$ is a branch of $\bigcup_{i<\omega} f_{1}\left(t_{i}\right)$. But, by the Claim below, $\bigcup_{i<\omega} f_{1}\left(t_{i}\right) \subset \bigcup_{x \in C} f_{1}(x) \in \mathrm{wf}(A)$, which is a contradiction. 
Claim. If $L \subset \mathrm{wf}(A)$ is compact, then $\bigcup L \in \mathrm{wf}(A)$.

Proof. Suppose $b \in A$ were a branch of $\bigcup L$. For every $n<\omega$, let $C_{n}=$ $\left\{T \in L:\left.b\right|_{n} \in T\right\}$. This is a decreasing sequence of nonempty closed subsets of $L$. By compactness, their intersection is nonempty, which implies that for some $T \in L, b$ is a branch of $T$.

Theorem 17. Let $A \subset \mathcal{N}$ and $\mathcal{T}=\bigcup_{a \in A} T_{a}$, let $\mathcal{F}=\mathcal{F}_{A}$ be the family of all closed and discrete subsets of $A$, and let $R[\mathcal{F}] \subset 2^{\mathcal{T}}$ be the compact set coming from an $(A, \mathcal{F})$-Reznichenko family of trees. Then there exists no determining function $f: \mathcal{T} \rightarrow A$.

For the proof we need Lemma 18 below, which is a generalization of $[1$, Lemma 6.2] with analogous proof. A subset $D$ of a tree $T$ is called successively dense if there is a countable family $R$ of immediate successors of the root such that every $t \in T$ incomparable with every element of $R$ has an immediate successor in $D$.

Lemma 18. Let $\left\{U_{n}\right\}_{n<\omega}$ be a disjoint family of open subsets of $A$ whose union is closed in $A$, and let $\mathcal{T}=\bigcup_{n<\omega} D_{n}$ be a countable decomposition of $\mathcal{T}$. Then there exists $n<\omega$ such that $D_{n} \cap T_{a}$ is successively dense in the tree $T_{a}$ for every $a \in U_{n}$.

Proof. Suppose that the statement is false. Then we can construct recursively a sequence $\left(a_{n}\right)$ of elements of $A$ and a sequence $\left(t_{n}\right)$ of elements of $\mathcal{T}$ with the following properties:

- $a_{n} \in U_{n}, t_{n} \in T_{a_{n}}$ for every $n$.

- No immediate successor of $t_{n}$ in $T_{a_{n}}$ belongs to $D_{n}$.

- The sets $S_{n}$ of predecessors of $t_{n}$ in $T_{a_{n}}$ are pairwise disjoint.

The construction is performed as follows: Assume that we have already defined $a_{i}$ and $t_{i}$ for $i<n$. From the negation of the lemma, we obtain $a_{n} \in U_{n}$ such that $D_{n} \cap T_{a_{n}}$ is not successively dense in $T_{a_{n}}$. Let $R$ be the set of those immediate successors of $a_{n}$ in $T_{a_{n}}$ which are comparable with some element of $\bigcup_{i<n} S_{i}$ in $T_{a_{n}}$. The set $R$ is finite. Hence, as $D_{n} \cap T_{a_{n}}$ is not successively dense in $T_{a_{n}}$, we can pick $t_{n} \in T_{a_{n}}$ incomparable with every $r \in R$ such that no immediate successor of $t_{n}$ belongs to $D_{n}$. This finishes the recursive construction.

Now, since $a_{n} \in U_{n}$ and the $U_{n}$ 's are disjoint open sets with closed union, the set $\left\{a_{n}: n<\omega\right\}$ is closed and discrete in $A$, hence it belongs to $\mathcal{F}$. From the definition of Reznichenko family of trees, we conclude that there must exist an element $t$ which is an immediate successor of the segment $S_{n}$ in $T_{a_{n}}$ for every $n$. For some $m, t \in D_{m}$. But this contradicts the assumption that $t_{m}$ has no immediate successor in $T_{a_{m}}$ belonging to $D_{m}$. 
Proof of Theorem 17. Suppose that there exists a determining function $f: \mathcal{T} \rightarrow A$. Remember that $A \subset \mathcal{N}$, and that for $s \in \omega^{<\omega}$ we have put $W_{s}=\{a \in A: s \prec a\}$. Let $D_{s}=f^{-1}\left(W_{s}\right)$, and let

$S_{0}=\left\{s \in \omega^{<\omega}: D_{s}\right.$ is successively dense in $T_{a}$ for every $\left.a \in W_{s}\right\}$

$S_{1}=\left\{s \in S_{0}: t \in S_{0}\right.$ for all $\left.t<s\right\}$,

$S_{2}=\left\{s \in \omega^{<\omega} \backslash S_{0}: s\right.$ is an immediate successor of some element of $\left.S_{1}\right\}$.

Notice that $S_{1}$ is a tree on $\omega$. We claim that $S_{1}$ has a branch $a \in A$. Indeed, if no branch of $S_{1}$ were an element of $A$, the union of the family $\left\{W_{s}: s \in S_{2}\right\}$ of disjoint clopen sets would be the whole $A$. We could then apply Lemma 18 to the decomposition $\mathcal{T}=\bigcup_{s \in S_{2}} D_{s}$ and the clopen sets $\left\{W_{s}: s \in S_{2}\right\}$ to conclude that there exists $s \in S_{2}$ such that $D_{s}$ is successively dense in $T_{a}$ for every $a \in W_{s}$, that is, $s \in S_{0}$, which contradicts $s \in S_{2}$.

Let $a \in A$ be a branch of $S_{1}$. Then $D_{a \mid n}$ is successively dense in $T_{a}$ for every $n<\omega$. Hence, for every $n<\omega$ there is a countable family $C_{n}$ of immediate successors of $a$ in $T_{a}$ such that every element of $T_{a}$ incomparable with $C_{n}$ has an immediate succesor in $D_{a \mid n}$. Let $t$ be an immediate successor of $a$ in $T_{a}$ such that $t \notin \bigcup_{n<\omega} C_{n}$. We can construct in $T_{a}$ an infinite sequence $t<_{a} t_{1}<_{a} t_{2}<_{a} \cdots$ with $t_{n} \in D_{a \mid n}=f^{-1}\left(W_{a \mid n}\right)$. This sequence is an element of $R[\mathcal{F}] \subset 2^{\mathcal{T}}$, contrary to $f$ being a determining function.

Let us recall the definition of the projective classes $\Sigma_{n}^{1}$ and $\Pi_{n}^{1}$. As already indicated, $\Sigma_{1}^{1}$ and $\Pi_{1}^{1}$ denote the classes of analytic and conalytic sets respectively. Recursively, $\Sigma_{n+1}^{1}$ is defined as the class of separable metrizable spaces which are continuous images of spaces in $\Pi_{n}^{1}$, and $\Pi_{n+1}^{1}$ are the separable metrizable spaces which are complements of sets in $\Sigma_{n+1}^{1}$ inside a Polish space.

Proof of Theorem 4. Let $A \subset \omega^{\omega} \times \omega^{\omega}$ be a universal $\Pi_{n-1}^{1}$ space, that is, a $\Pi_{n-1}^{1}$ set such that for every $\Pi_{n-1}^{1}$ subset $B$ of $\omega^{\omega}$ there exists $b \in \omega^{\omega}$ such that $\{b\} \times B=A \cap\left(\{b\} \times \omega^{\omega}\right)$. Such a set always exists (cf. [7]). Set $X=C\left(R\left[\mathcal{F}_{A}\right]\right)$. By Theorem 17 , for $R\left[\mathcal{F}_{A}\right] \subset 2^{\mathcal{T}}$, there is no determining function $f: \mathcal{T} \rightarrow A$. Actually, this implies that there is no determining function to any $\Sigma_{n}^{1}$ set, in view of Lemma 8 and the fact that $A$ maps continuously onto any $\Sigma_{n}^{1}$ set.

Finally, we prove that $C\left(R\left[\mathcal{F}_{A}\right]\right)$ is weakly $\Sigma_{n+1}^{1}$-determined. By Theorem 16, it is enough to show that $\mathrm{wf}(A)$ belongs in $\Pi_{n}^{1} \subset \Sigma_{n+1}^{1}$. Put

$$
\begin{aligned}
& S=\{x \in \operatorname{Tr}: \exists a \in A \text { which is a branch of } x\}, \\
& \Omega=\{(x, a) \in \operatorname{Tr} \times A: a \text { is a branch of } x\} .
\end{aligned}
$$

Then $\Omega$ is a closed subset of $\operatorname{Tr} \times A$, hence a $\Pi_{n-1}^{1}$ set like $A$. The set $S$ is the projection of $\Omega$ to the first coordinate, so $S$ is $\Sigma_{n}^{1}$. Finally, wf $(A)=\operatorname{Tr} \backslash S$ is $\Pi_{n}^{1}$. 
Acknowledgements. I wish to thank Ondřej Kalenda for his useful remarks concerning the work of Čižek and Fabian, and also Pandelis Dodos for teaching me the descriptive set-theoretic way of thinking which is somewhat present in this note. An acknowledgement is also due to the referee for a number of corrections and improvements in the final form of the article.

\section{References}

[1] S. A. Argyros, A. Arvanitakis and S. Mercourakis, Talagrand's $K_{\sigma \delta}$ problem, preprint, 2007.

[2] S. A. Argyros and V. Farmaki, On the structure of weakly compact subsets of Hilbert spaces and applications to the geometry of Banach spaces, Trans. Amer. Math. Soc. 289 (1985), 409-427.

[3] P. Čížek and M. Fabian, Adequate compacta which are Gul'ko or Talagrand, Serdica Math. J. 29 (2003), 55-64.

[4] M. Fabian, Gâteaux Differentiability of Convex Functions and Topology, Canad. Math. Soc. Ser. Monogr. Adv. Texts, Wiley, New York, 1997.

[5] M. Fabian, G. Godefroy, V. Montesinos and V. Zizler, Inner characterizations of weakly compactly generated Banach spaces and their relatives, J. Math. Anal. Appl. 297 (2004), 419-455.

[6] V. Farmaki, The structure of Eberlein, uniformly Eberlein and Talagrand compact spaces in $\Sigma\left(R^{\Gamma}\right)$, Fund. Math. 128 (1987), 15-28.

[7] A. S. Kechris, Classical Descriptive Set Theory, Grad. Texts in Math. 156, Springer, New York, 1995.

[8] S. Mercourakis, On weakly countably determined Banach spaces, Trans. Amer. Math. Soc. 300 (1987), 307-327.

[9] M. Talagrand, Espaces de Banach faiblement $\mathcal{K}$-analytiques, Ann. of Math. (2) 110 (1979), 407-438.

[10] -, A new countably determined Banach space, Israel J. Math. 47 (1984), 75-80.

[11] L. Vašák, On a generalization of weakly compactly generated spaces, Studia Math. 70 (1981), 11-19.

Équipe de Logique Mathématique

UFR de Mathématiques

Université de Paris 7

2 Place Jussieu

75251 Paris, France

E-mail: avileslo@um.es

Received June 26, 2007

Revised version February 10, 2008

(6181) 